\title{
CONFIGURATION AND PERFORMANCE ANALYSIS OF DEEP OCEAN MINING FLEXIBLE RISER
}

\author{
Yuxin Gai\# \\ School of Aeronautics Sciences and \\ Engineering, Beihang University \\ Beijing, China \\ Yilun Li \\ Sino-French Engineer School, Beihang \\ University, Beijing, China \\ School of Aeronautics Sciences and \\ Engineering, Beihang University
}

\author{
Shuangxi Guo\# \\ Institute of Mechanics, Chinese Academy of \\ Sciences \\ School of Engineering Science, University of \\ Chinese Academy of Sciences, Beijing, China \\ Min Li \\ School of Aeronautics Sciences and Engineering, \\ Beihang University, Beijing, China
}

\section{Weimin Chen* \\ Institute of Mechanics, Chinese Academy of Sciences \\ School of Engineering Science, University of Chinese Academy of Sciences}

Beijing, China

\section{ABSTRACT}

Deep-sea minerals such as polymetallic nodule, hydrothermal sulphides and ferro-manganese crusts have for long attracted attention as an alternative source of metals to terrestrial deposits. To bring these minerals up to the land, flexible risers are needed. As the mining industry developing towards deep sea area, the conveying system is usually designed as a combination of steel riser and flexible riser. According to different transport requirements, various flexible riser configurations, such as steel catenary riser, lazy-wave riser and saddle-shaped riser, have been proposed. During mining operation, the riser bears gravity, buoyancy, wave and current force, therefore the assessment of structural safety and reliability is quite challenging. In addition, the riser response caused by the mining vehicle motion during working process in a large area should also be considered. To guarantee a safe operation and service life of the riser, it is necessary to carefully design its configuration and to analyze its performance.

In this study, taking the saddle-shaped riser as our model, the influences of main design parameters on the riser configuration, tension and stress are examined. These parameters include the installation position of buoyancy modules, the buoyancy ratio and motion of mining vehicle. Firstly, the analysis model of the riser response is established based on FEM in which the nonlinear large displacement and deformation of the structure are considered. Secondly, through our FEM simulation, the distribution and variation of tension and stress along the axial length of risers with different configurations are presented. Finally, the impacts of the mining vehicle motion on riser response are discussed. Our numerical results show that a small change of the buoyancy position and buoyancy ratio may lead to a significant change of the riser configuration, but a little change of riser tension/stress. And the saddle-shaped riser has a good tolerance performance to the bottom-end excitation.

Keywords: Deep-sea mining; flexible riser; riser configuration; response and performance

$\begin{array}{cl}\text { NOMENCLATURE } \\ U_{e} & \text { element displacement vector } \\ u_{i} & \text { axial displacement } \\ v_{i} & \text { lateral displacement } \\ \theta_{i} & \text { rotational displacement } \\ l_{e} & \text { element length } \\ K_{e} & \text { element stiffness matrix } \\ N_{i} & \text { shape function } \\ B_{i} & \text { strain matrix } \\ x_{e} & \text { axial location }\end{array}$

* Contact author: wmchen@imech.ac.cn

\# These authors contributed equally to this work. 


$\begin{array}{ll}\xi & \text { dimensionless location } \\ E & \text { elastic modulus of riser } \\ {[K]} & \text { riser stiffness matrix } \\ {[U]} & \text { riser displacement vector } \\ {[P]} & \text { load vector } \\ {[\sigma]} & \text { stress vector } \\ {[B]} & \text { strain matrix of the riser } \\ L_{B} & \text { distribution position of Buoyancy B } \\ F_{B} & \text { equivalent buoyancy force of Buoyancy B } \\ L_{C} & \text { distribution position of Buoyancy C } \\ F_{C} & \text { equivalent buoyancy force of Buoyancy C }\end{array}$

\section{INTRODUCTION}

With the development of offshore oil and gas production and mining industry, riser systems of various configurations have been used for oil/gas/brine transportation, and they are becoming an important part of the whole production system. These riser systems usually include top tension riser (TTR), steel catenary riser (SCR) and hybrid riser [1]. With the increase of water depth, tension on the upper-end of SCR increases rapidly, which limits its further application in deep waters [2]. The TTR may suffer from a large tension due to the motion of the top-end float under environmental loads, while hybrid riser's joint is one of the difficult problems to deal with. Therefore, some new riser configurations have been proposed to meet the requirements for deep water production, e.g. compliant vertical access risers and steel lazy-wave riser [3-5] for oil/gas platform, and saddle shape riser [6] for ocean mining systems.

To ensure the normal operation and a longer service life of riser system, its configuration needs to be designed carefully based on comprehensive examination of its structural response, such as the tension, stress and displacement of riser under excitation of environmental loads and moving boundary conditions. For SCR, TTR and lazy-wave riser, a large number of researches on riser design and response analysis are available for reference. Dong[7] and Lan[8] studied the fatigue response of a SCR in the touchdown zone and evaluated the mechanical behavior and the global performance of the riser. Some studied the dynamic response of the SCR under the influence of the internal flow[9], the vessel motion[10] and other factors. Chatjigeorgiou[11] investigated the effect of the steady flow inside the pipe on both the in-plane and the out-of-plane vibrations through his numerical simulations. Ai[12] studied the stress and fatigue damage along the lazy-wave risers with different distribution of buoyancy modules using genetic algorithms. Guo[13] studied the influences of the amplitude/frequency of the top vessel motion, along with the buoyancy modules distribution along structural length, on dynamic responses of lazy-wave and hybrid-tower types of risers. Jang[14] studied VIV(vortex-induced vibration) response of a long flexible lazy-wave riser using CFD simulations and $\mathrm{Li}[15]$ studied the multi-frequency vortex-induced vibration of a TTR in lineally sheared fluid field using the numerical approach. However, for case of a saddle-shaped riser, there have been few reports on structural response, particularly for a mining system in deep sea.
For deep water field development, different types of flexible risers with a series of buoyancy modules are developed. Both the configuration and the mean tension level of the riser, which is mainly induced by the riser self-weight are strongly affected by the distribution of buoyancy modules. Before practical application, the buoyancy force and installation position of the modules should be carefully designed to archive a good performance. A high tension level may lead extremely restriction on the top-end float and/or bottom-end vehicle motions amplitudes to ensure limited amplitude of the riser tension variation range. On the other hand, the motion of top-end float and/or bottom-end vehicle is strictly controlled to prevent inducing excessive curvature and compression in riser.

In this study, to improve the riser's tension level and operational performance, the preliminary design analysis of a saddle-shaped riser is carried out through the finite element simulations. Firstly, the influences of the installation position of buoyancy modules and the buoyancy ratio (the ratio of buoyancy $\mathrm{B}$ and buoyancy $\mathrm{C}$ as shown in Fig.1) on the configuration, as well as tension and stress of the saddle-shaped riser, are studied. Further, the sensitivity of riser's key parameters to the motion of mining vehicle is analyzed under different installation scheme of buoyancy modules. The results show that the installation position of buoyancy modules would cause significant changes of riser configuration. Then the buoyancy ratio of the two buoyancy modules could also effectively reduce the maximum tension and the top tension of the riser. Finally, it is found that the saddle-shaped riser has a good response performance to the bottom-end excitation caused by the mining vehicle.

\section{Riser Model}

\subsection{Riser Parameters and Load Cases}

The main parameters of the flexible riser considered in this study is selected according to the Project of the Deep-Ocean Mining System with $1 \mathrm{~km}$ water depth in the South Chinese Sea. The riser has a special configuration of saddle shape as shown in Fig.1, and its total length is $400 \mathrm{~m}$. The main parameters [6] are listed in Table 1.

The responses, including the configurations, tensions and stress responses of the riser under different load cases, are calculated, where the location of buoyancy modules, buoyancy ratio and the miner position vary in different cases. The buoyancy locations and buoyancy forces (buoyancy ratio) in different cases are summarized in Table 2. The distribution length of Buoyancy B and C are both $20 \mathrm{~m}$ and keeps constant in this study. $L_{B}$ indicates the distribution position of Buoyancy B, and $F_{B}$ is the equivalent buoyancy force. $L_{C}$ and $F_{C}$ represent the buoyancy position and force of Buoyancy $\mathrm{C}$, and the buoyancy ratio is defined as $F_{B} / F_{C}$. The riser length is measured from the upper-end A to the bottom-end D. Through our calculations of riser response for Cases 1-4, the influences of the installation position of buoyancy modules on riser configuration, tension and stress will be studied. The comparisons between Case 4, Case 5 and Case 6 will show the influence of the buoyancy ratio on the riser performance during operating state. 


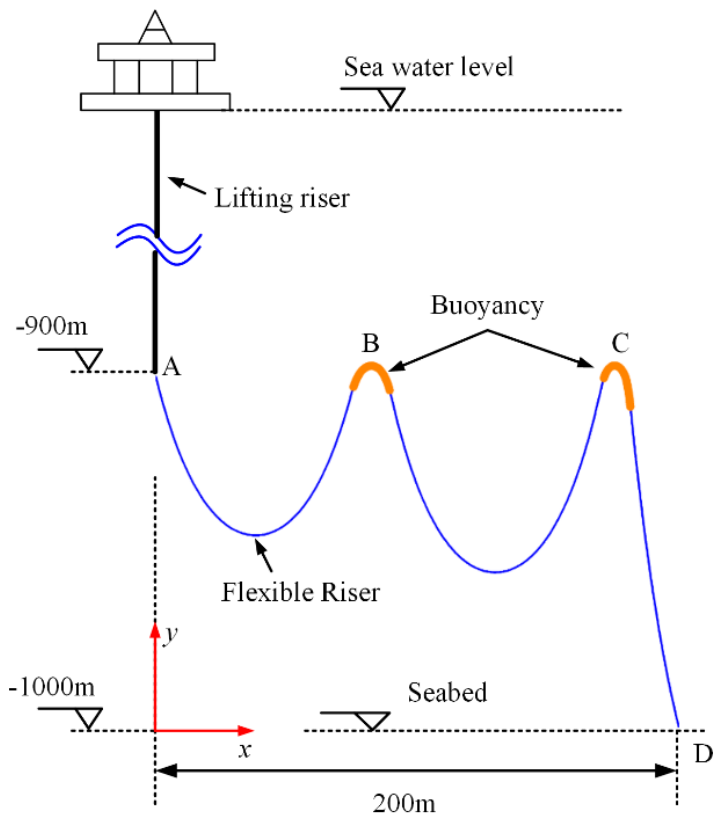

FIGURE 1: CONFIGURATION OF THE SADDLE-SHAPED FLEXIBLE RISER

TABLE 1. PARAMETERS OF THE FLEXIBLE RISER

\begin{tabular}{cc}
\hline Parameter & Value \\
\hline Outside diameter & $205.0 \mathrm{~mm}$ \\
Inner diameter & $150.0 \mathrm{~mm}$ \\
Length & $400 \mathrm{~m}$ \\
Weight in water & $30.0 \mathrm{~kg} / \mathrm{m}$ \\
Tensile stiffness & $1.536 \mathrm{e} 9 \mathrm{~N}$ \\
Bending stiffness & $2.096 \mathrm{e} 4 \mathrm{~N} \cdot \mathrm{m}^{2}$ \\
\hline
\end{tabular}

TABLE 2. LOCATION OF BUOYANCY AND BUOYANCY FORCE IN DIFFERENT CASES

\begin{tabular}{ccccc}
\hline Cases & $L_{B}$ & $F_{B}$ & $L_{C}$ & $F_{C}$ \\
\hline 1 & $123 \mathrm{~m}-143 \mathrm{~m}$ & $4.0 \mathrm{t}$ & $256 \mathrm{~m}-276 \mathrm{~m}$ & $8.0 \mathrm{t}$ \\
2 & $123 \mathrm{~m}-143 \mathrm{~m}$ & $4.0 \mathrm{t}$ & $266 \mathrm{~m}-286 \mathrm{~m}$ & $8.0 \mathrm{t}$ \\
3 & $123 \mathrm{~m}-143 \mathrm{~m}$ & $4.0 \mathrm{t}$ & $276 \mathrm{~m}-296 \mathrm{~m}$ & $8.0 \mathrm{t}$ \\
4 & $123 \mathrm{~m}-143 \mathrm{~m}$ & $4.0 \mathrm{t}$ & $286 \mathrm{~m}-306 \mathrm{~m}$ & $8.0 \mathrm{t}$ \\
5 & $123 \mathrm{~m}-143 \mathrm{~m}$ & $4.25 \mathrm{t}$ & $286 \mathrm{~m}-306 \mathrm{~m}$ & $7.75 \mathrm{t}$ \\
6 & $123 \mathrm{~m}-143 \mathrm{~m}$ & $4.5 \mathrm{t}$ & $286 \mathrm{~m}-306 \mathrm{~m}$ & $7.5 \mathrm{t}$ \\
\hline
\end{tabular}

\subsection{FEM Model}

The finite element simulation[16] is used in this study to calculate the response of the riser. The whole riser is divided into a set of beam elements, since the motion of the riser is in the $x-y$ plane (see Fig.1). Only the in plane displacements are considered for each element (Fig.2), the displacement vector of the beam element is

$$
U_{e}=\left[\begin{array}{llllll}
u_{i} & v_{i} & \theta_{i} & u_{i+1} & v_{i+1} & \theta_{i+1}
\end{array}\right]^{T}
$$

where $u$ and $v$ are the axial and translational displacements respectively. $\theta$ is the rotational angle, and $i$ is the node number of the beam element. For simplicity, the displacement vector is divided into two parts, i.e. the axial part and the lateral part

$$
\begin{gathered}
U_{e}^{1}=\left[\begin{array}{ll}
u_{i} & u_{i+1}
\end{array}\right]^{T} \\
U_{e}^{2}=\left[\begin{array}{llll}
v_{i} & \theta_{i} & v_{i+1} & \theta_{i+1}
\end{array}\right]^{T}
\end{gathered}
$$

The shape functions are

$\mathbf{N}_{1}\left(x_{e}\right)=\left[1-x_{e} / l_{e}, x_{e} / l_{e}\right]$

$\mathbf{N}_{2}\left(x_{e}\right)=\left[1-3 \xi^{2}+2 \xi^{3}, l_{e}\left(\xi-2 \xi^{2}+\xi^{3}\right), 3 \xi^{2}-2 \xi^{3}, l_{e}\left(\xi^{3}-\xi^{2}\right)\right]$

where $\xi=x_{e} / l_{e}$ and $x_{e}$ are the axial location. The strain matrix can be written as

$$
B_{i}\left(x_{e}\right)=\frac{d\left(N_{i}\right)}{d\left(x_{e}\right)}
$$

where $A_{e}$ is the cross area. Then the stiffness of the element can be written as

$$
\begin{aligned}
K_{e}^{l} & =\int_{0}^{l_{e}} \int_{A_{e}} B_{1}^{T} E_{e} B_{l} d A_{e} d x_{e} \\
K_{e}^{2} & =\int_{0}^{l_{e}} \int_{A_{e}} B_{2}^{T} E_{e} B_{2} d A_{e} d x_{e}
\end{aligned}
$$

Assembling the stiffness matrix in Eq.(4), we can obtain the element stiffness matrix $K_{\mathrm{e}}$, and, subsequently, the whole structural matrices. The static governing equation of the flexible riser is

$$
[K] \cdot[U]=[P]
$$

where $[K],[U]$ and $[P]$ are the stiffness matrix, displacement vector and load vector of the whole riser respectively. Solving Eq.(6), we can obtain the displacement vector $[U]$ of the riser. And the average stress vector $[\sigma]$ can be obtain with the following equation

$$
[\sigma]=E \cdot[B] \cdot[U]
$$

where, $E$ is the elastic modulus of the riser and $[B]$ is the strain matrix. Once we got the stress distribution, the tension can be obtain by multiply the stress and the cross section area.

Here, the stress vector $[\sigma]$ calculated using Eq.(7) is the average stress at the cross section, and the bending stress $\sigma_{B}$ can be obtained by the following equation

$$
\sigma_{B}=E \frac{y}{\rho}
$$

where, $\rho$ is the curvature radius of the riser and $y$ is the distance from point on cross section to neutral axis. And the relationship between the radius of curvature and the structural displacement is

$$
\frac{1}{\rho}=\frac{d^{2}[U]}{d x^{2}}
$$

Using Eq.(7)-(9) we can obtain the bending and axial stresses of the riser.

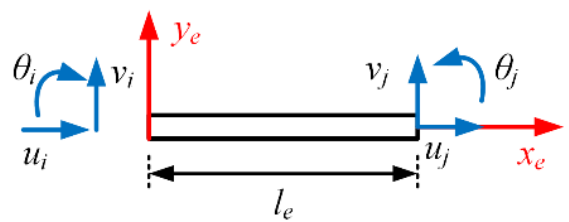

FIGURE 2: BEAM ELEMENT IN LOCAL COORDINATE SYSTEM 


\subsection{Model Verification}

The FEM model was developed here to calculate the tension, stress, displacement along with the configuration of the flexible riser. To verify the FEM model, the configuration and tension of a catenary mooring-line is calculated and compared with the experimental results. The parameters of the catenary are listed in Table 3[17], and Fig.3 (a) shows a schematic diagram of the structure.

TABLE 3. PARAMETERS OF THE SECOND CATENARY MOORING-LINE

\begin{tabular}{cc}
\hline Parameter & Value \\
\hline Total length & $21.0 \mathrm{~m}$ \\
Initial vertical projection & $5.0 \mathrm{~m}$ \\
Axial stiffness & $3.4 \mathrm{e} 5 \mathrm{~N}$ \\
Equivalent hydrodynamic diameter & $0.0034 \mathrm{~m}$ \\
Mass per unit length & $0.069 \mathrm{~kg} / \mathrm{m}$ \\
Wet weight per unit length & $0.5872 \mathrm{~N} / \mathrm{m}$ \\
\hline
\end{tabular}

Firstly, two catenary configurations with different horizontal projection, i.e. $19.872 \mathrm{~m}$ for Configuration 1 and $19.364 \mathrm{~m}$ for Configuration 2 are calculated and compared with the experimental profiles. The comparison of catenary configurations is shown in Fig.3 (b). Table 4 shows the difference between the computed tension at the fairlead and the experimental measurement for both configurations, the difference in the predicted static tension is below $3 \%$. It can be seen that both the tension and configurations agree well with the experimental results.

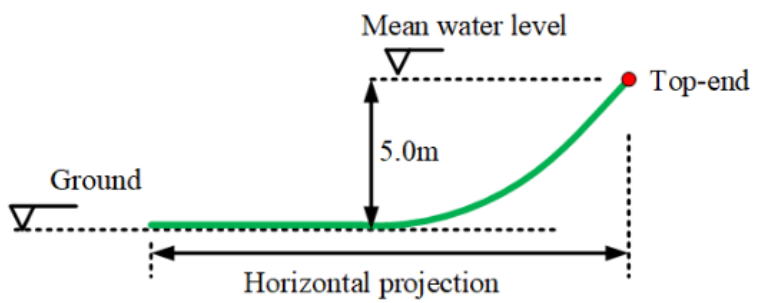

(a)

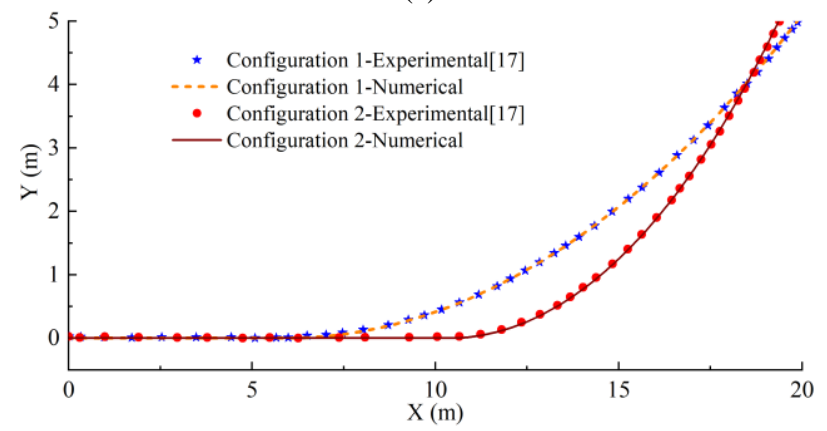

(b)

FIGURE 3: COMPARISON OF NUMERICAL AND EXPERIMENTAL RESULTS (a) SCHEMATIC OF THE EXPERIMENTAL CATENARY (b) CONFIGURATIONS OF THE CATENARY

TABLE 4. COMPARISON OF STATIC FAIRLEAD TENSION

\begin{tabular}{cccc}
\hline Configuration & Experimental/N[17] & Numerical/N & difference \\
\hline 1 & 14.48 & 14.82 & $2.35 \%$ \\
2 & 8.13 & 8.11 & $0.25 \%$ \\
\hline
\end{tabular}

\section{Numerical Results}

The structural safety and performance of a riser, in practical applications, are directly related to the level of riser tension/stress during mining operation, so it is necessary to design riser configuration carefully to ensure the safety and reliability of riser structure. A reasonable arrangement of buoyancy modules position and the buoyancy ratio in different regions is the important concerns of configuration design. On the other hand, the motion of the bottom mining vehicle could also cause a change of structural configuration and tension. In this section, the riser responses caused by different installation location of buoyancy modules, buoyancy ratios and motions of mining vehicle are calculated. During our calculations, it is regarded that both ends of the riser are hinged supports, and the riser motion is in the $x$ - $y$ plane, so the static 2D beam simulation is used as shown in Section 1.2.

\subsection{Buoyancy Module Location}

To analyze the influence of buoyancy module location on riser structure response, the configuration, tension and stress of the risers for Cases1-4 are calculated and compared with each other. Configuration comparison is shown in Fig.4.

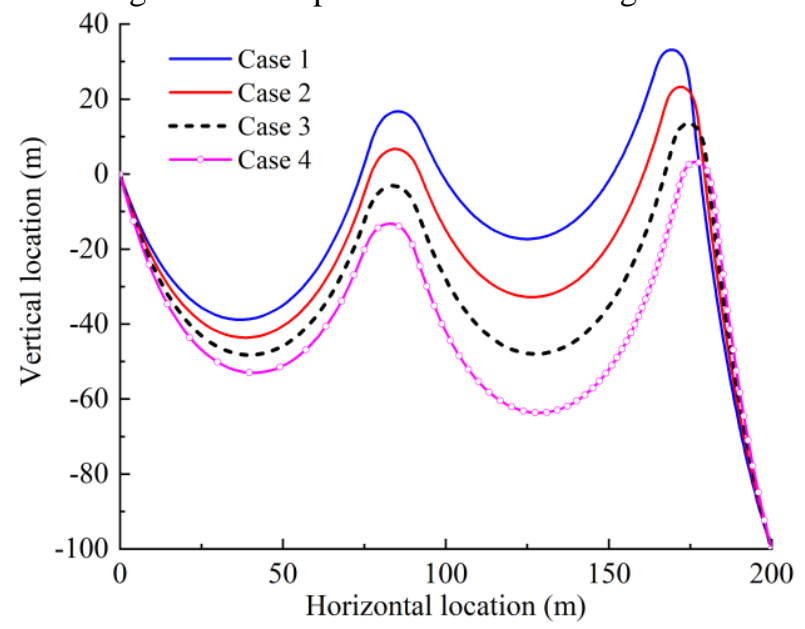

FIGURE 4: COMPARISON OF CONFIGURATIONS IN CASE 1CASE 4

It can be seen that the position of buoyancy module has a significant influence on riser configuration. As $L_{C}$ gets larger, i.e with the movement of Buoyancy $\mathrm{C}$ down toward the bottom-end, the overall displacement of the riser gradually drops. With the movement of the buoyancy module down toward the end, much buoyancy force is distributed on the part of segment $\mathrm{CD}$ of the riser (Fig.1). The decrease in buoyancy of AC segment provided by the buoyancy module is mainly responsible for the descending of the riser equilibrium position. On the other hand, positioning of the buoyancy module down toward bottom-end can also cause the increase of tension at the two ends.

Figure 5 shows the distribution of structural tension along riser length for different load cases. It can be seen that the maximum tension, $53 \mathrm{kN}$, occurs near Buoyancy $\mathrm{C}$, which is much higher than the tension at Buoyancy $\mathrm{B}, 20 \mathrm{kN}$. With the movement of the buoyancy $\mathrm{C}$ down toward the bottom-end, the 
maximum tension tends to decrease, and the maximum tension in Case 4 decreases by about 9\% compared with Case 1 . However, the tension of riser's two ends tends to increase. It is worthwhile to note that the tension value of the bottom-end should be designed to keep within a designed range, or it should not exceed the value of the gravity of the bottom mining vehicle.

The bending stress distributions of the riser under the 4 cases are shown in Fig.6. Different from the tension, the bending stress increases firstly and then decreases, while it does not significantly change with the variation of buoyancy position.

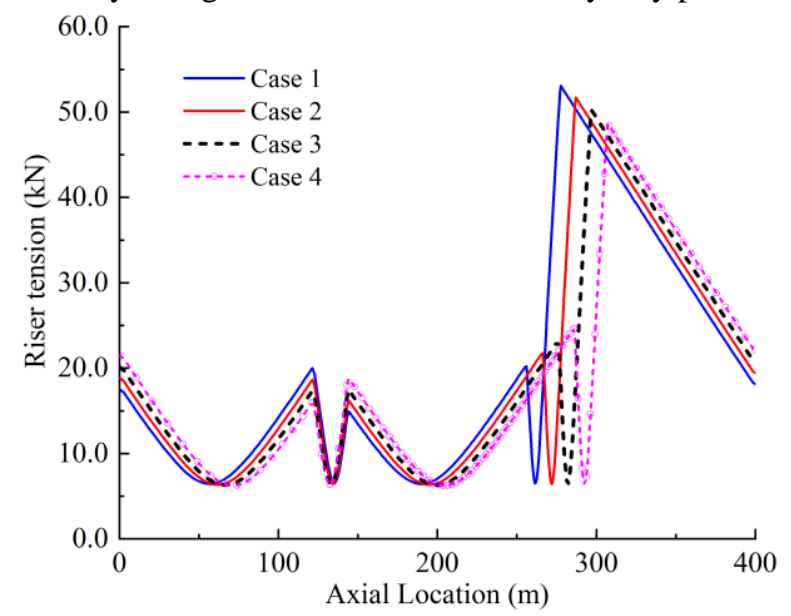

FIGURE 5: COMPARISON OF RISER TENSION DISTRIBUTION IN CASE 1-CASE 4

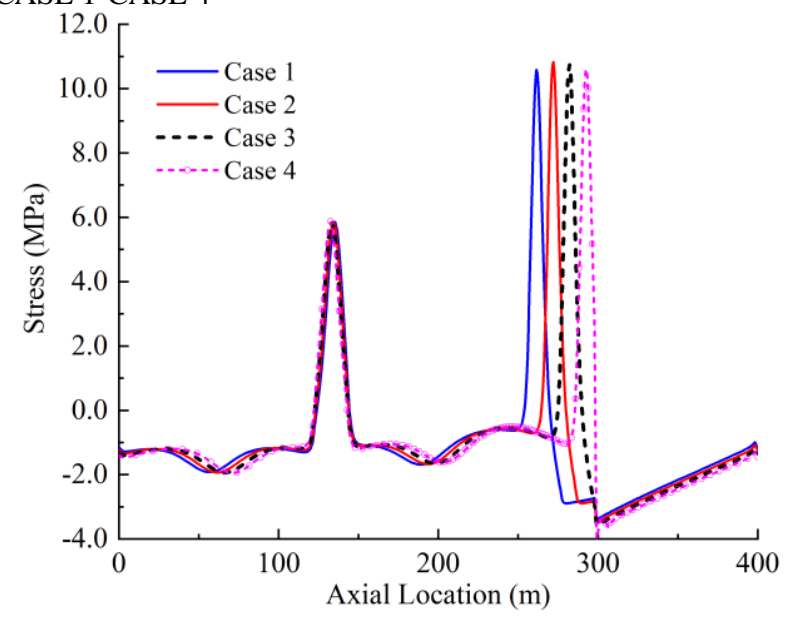

FIGURE 6: COMPARISON OF RISER STRESS DISTRIBUTION IN CASE 1-CASE 4

\subsection{Buoyancy Ratio}

Buoyancy ratio of the two buoyancy modules is also one of the important factors that affect riser configuration and response. The influences of buoyancy ratio on riser configuration, tension and stress are examined, while the position and total buoyancy force remain the same. The riser configurations under different operation conditions, i.e. Cases 4-6, are plotted in Fig.7. It can be seen that the change of buoyancy ratio has a significant impact on the overall configuration. In the three load cases, the most obvious change of riser position happens at the location of buoyancy B. The vertical position gets larger by approximately
$16 \mathrm{~m}$ for every $0.25 \mathrm{t}$ increase of buoyancy force. However, the change of buoyancy ratio has no obvious influence on the riser position at the location of buoyancy $\mathrm{C}$. Because the bottom-end is always in a tensional state, while the change of buoyancy can only affect the pre-tension. This can be further proven in the tension plots presented in Fig. 7.

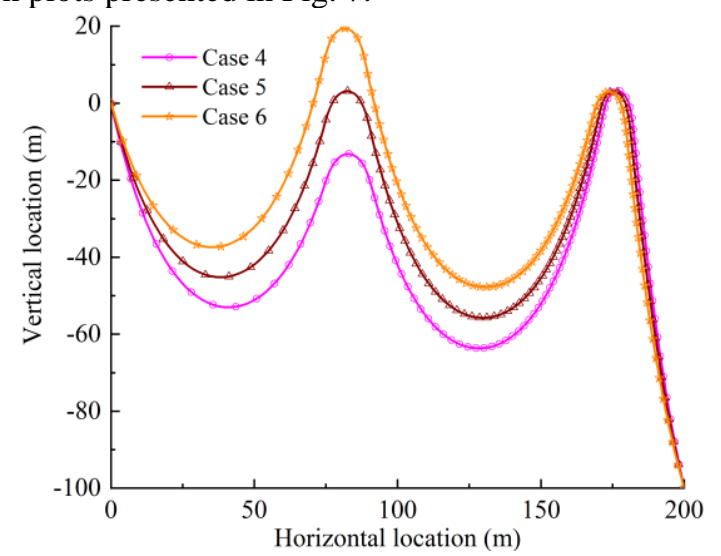

FIGURE 7: COMPARISON OF CONFIGURATIONS IN CASE 4CASE 6

The tension distributions along riser length, with different buoyancy ratio, are shown in Fig.8. The maximum tension at the two-end tension decrease with the increase of buoyancy ratio. That is mainly because the decrease of $F_{C}$ could reduce the pretension at the bottom-end, while the increase of $F_{B}$ could make a larger buoyancy to balance with the structural gravity. That reduce the value of gravity required to be balanced by the constraint force component of the upper-end, or the tension at the upper-end decreases. Compared with the maximum tension of $48.36 \mathrm{KN}$ in Case 4, the maximum tension in Case 6, 43.47KN, decreases by about $10 \%$. The tension at the upper-end reduces from $21.49 \mathrm{KN}$ to $16.78 \mathrm{KN}$, by about $22 \%$.

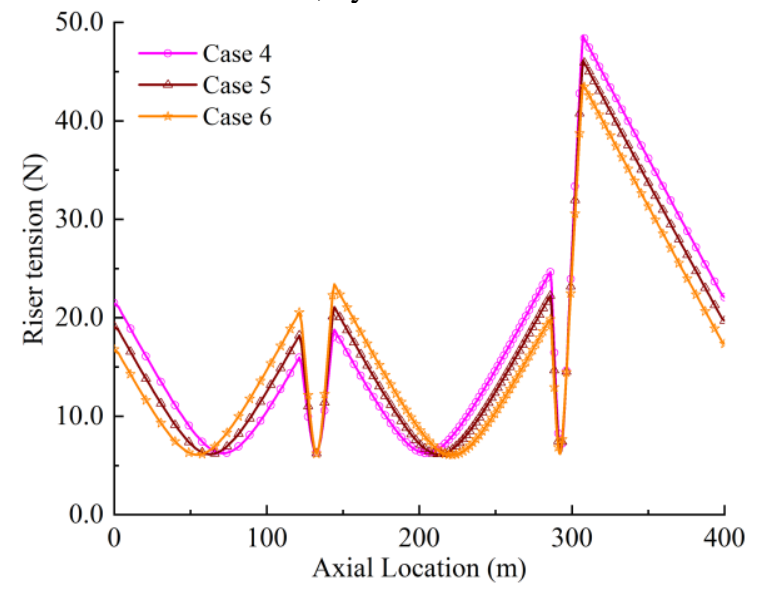

FIGURE 8: COMPARISON OF RISER TENSION DISTRIBUTION IN CASE 4-CASE 6

Fig.9 shows the distributions of the bending stress along the riser length for the three load cases. With the change of buoyancy ratio, the bending stress at the location of buoyancy $\mathrm{C}$ change little, since the shape of the riser dos almost not change (Fig.7). With the increase of buoyancy ratio, the bending stress at the location position of buoyancy B presents a slight increase. 


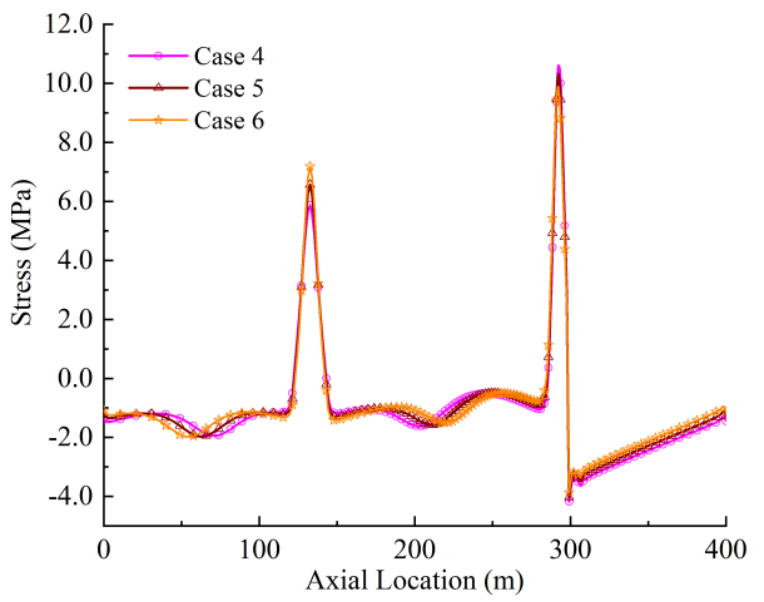

FIGURE 9: COMPARISON OF RISER STRESS DISTRIBUTION IN CASE 4-CASE 6

\subsection{Mechanical Performance in different positions of mining vehicle}

For given structural length and buoyancy module position of a riser, the motion of mining vehicle will directly changes the bottom-end position of the riser, that is to say that the horizontal projection of the riser may get larger or smaller. As the length of and the vertical projection of the riser remain the same, the variation of the horizontal projection will cause the change of its configuration. And consequently, the curvature of riser and the angles at the top and bottom ends become different, lead to the changes of axial tension and stress. The motion range of mining vehicle should be considered comprehensively during riser operation design. The riser responses are calculated when the mining vehicle operates in different position, for 3 load cases respectively. Here, the far-position (i.e. Far, horizontal projection is $250 \mathrm{~m}$ ) was defined as drift away from the upper-end (Fig.10), while the intermediate position was set as the equilibrium position (i.e. Neutral, horizontal projection is $200 \mathrm{~m}$ ), and the nearposition was defined as the position when offset was closest to the upper-end (i.e. Near, horizontal projection is $150 \mathrm{~m}$ ).

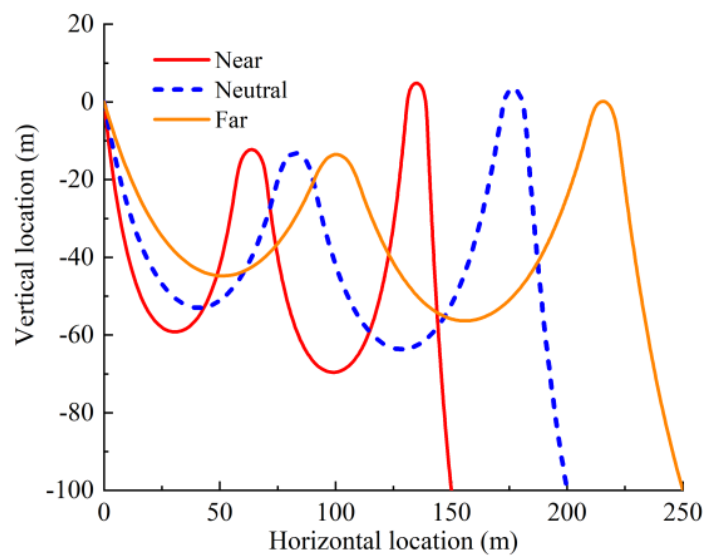

FIGURE 10: RISER CONFIGURATIONS WITH DIFFERENT MINING VEHICLE POSITION
Fig.11 shows the change of the maximum tension, for six load cases, when the mining vehicle is in different positions. Compared with the neutral-position, the maximum tension decreases by $0.46 \mathrm{KN}$, when the mining vehicle move to the nearposition. While the maximum tension increases by $1.23 \mathrm{KN}$ when the mining vehicle moves to the far-position. This change of maximum tension indicates that the motion of mining vehicle to the far-position has a bigger influence on the riser tension. On the other hand, if concerning the absolute value of the tension change, the influence of mining vehicle motion is not obvious.

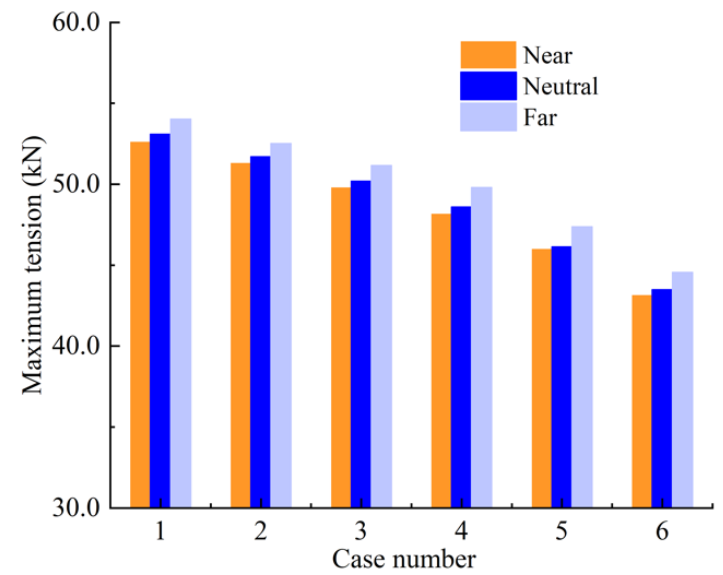

FIGURE 11: COMPARISON OF MAXIMUM RISER TENSION IN DIFFERENT POSITION

Fig.12 shows the change of the maximum riser stress, for the six load cases, when the mining vehicle is in different positions. It can be seen that the change of mining vehicle position has a significant impact on the maximum stress. When the mining vehicle moves to the near-position, the maximum stress increases by about $18.0 \%$. And, when it moves to the farposition, the maximum stress decreases by about $20.0 \%$. For the six load cases, the variations of riser stress caused by the mining vehicle position are almost the same, which means that the saddle-shaped riser has a good tolerance performance to the bottom-end excitation.

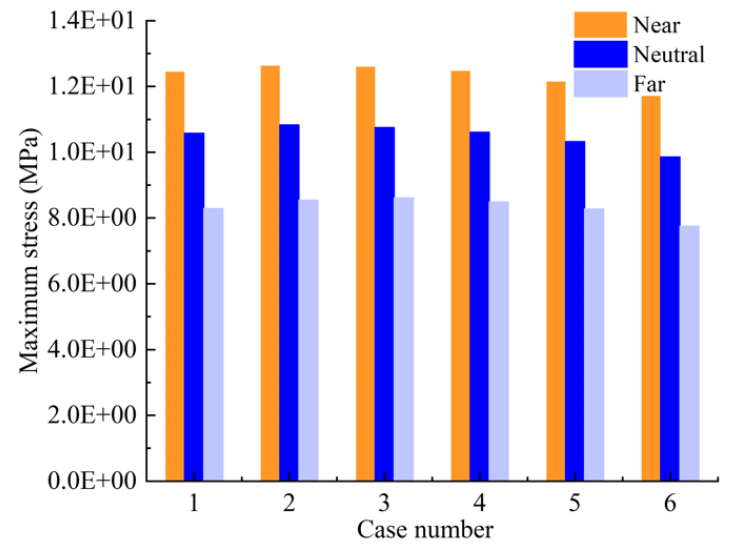

FIGURE 12: COMPARISON OF MAXIMUM RISER STRESS IN DIFFERENT POSITION 


\section{CONCLUSION}

The response analysis model of saddle-shaped mining riser is developed based on finite element simulation. By examining the tension and stress distributions along the riser length, the influences of some main factors, i.e. the buoyancy position, the buoyancy ratio and the position of the mining vehicle, on the structural responses are studied, in terms of the tension, stress and configuration of the riser.

The numerical results show that the buoyancy position and buoyancy ratio have significant impacts on the riser configuration and tension. For examples, the changes of the buoyancy module position could introduce a $46 \mathrm{~m}$ decrease of the riser vertical position. A small change of the buoyancy ratio can reduce the maximum tension and the top tension respectively by about $10 \%$ and up to $22 \%$. The variations of riser stress caused by the mining vehicle position for 6 cases are almost the same, indicates that the saddle-shaped riser has a good tolerance performance to the bottom-end excitation.

In this study, to improve the riser's tension level and operational performance, the preliminary design analysis of a saddle-shaped riser is carried out through the finite element simulations. Or, our focus is the global/static response of the saddle-shaped riser. More detailed/further work, such as local stress, dynamic responses and VIV, will be carried out in the future. And, the hydrodynamic loads caused by the external and/or internal flow would be considered.

\section{ACKNOWLEDGEMENTS}

The authors of this paper would like to thank the financial supports provided by the Strategic Priority Research Programme of the Chinese Academy of Sciences (Grant No. XDA22000000).

\section{REFERENCES}

[1] Lou M, Hu P, Qi X, et al. Stability analysis of deepwater compliant vertical access riser about parametric excitation[J]. International Journal of Naval Architecture and Ocean Engineering, 2019, 11:970-979.

[2] Wang J, Duan M. A nonlinear model for deepwater steel lazy-wave riser configuration with ocean current and internal flow[J]. Ocean Engineering, 2015, 94:155-162.

[3] Santillan S T, Virgin L N. Numerical and experimental analysis of the static behavior of highly deformed risers[J]. Ocean Engineering, 2011, 38(13):1397-1402.

[4] Pearce JL, Sizer PS, Gano JC, Yonker JH, Thurman RL, O'Sullivan JF, et al. Method and system for maintenance and servicing of subsea wells. United States Patent no. 4,730,677; March 1988.
[5] Tian Y, Hou Y, Pires F, et al. Tensioned Step Riser Configuration for Ultra-Deep Application[C], ASME 2015, International Conference on Ocean, Offshore and Arctic Engineering. 2015:V05AT04A056.

[6] Wang G, Liu S J, Li L. FEM modeling for 3D dynamic analysis of deep-ocean mining pipeline and its experimental verification $[\mathrm{J}]$. Journal of Central South University, 2007, 14(6):808-813.

[7] Dong X, Shiri H. Performance of non-linear seabed interaction models for steel catenary risers, part II: Global response[J]. Ocean Engineering, 2019, 82:158-174.

[8] Lan S. Numerical simulation of the mechanical interactions between the deepwater steel catenary riser and the touchdown zone of seabed[J]. Chinese Journal of Applied Mechanics, 2019,36(6):1478-1483.

[9] Liu Z, Guo H. Dynamic Response Study of Steel Catenary Riser Based on Slender Rod Model[J]. China Ocean Engineering, 2019, 33(1):57-64.

[10] Wang J, Fu S, Baarholm R, et al. Global motion reconstruction of a steel catenary riser under vessel motion[J]. Ships and Offshore Structures, 2019, 14(5):442-456.

[11] Chatjigeorgiou I K. Three dimensional nonlinear dynamics of submerged, extensible catenary pipes conveying fluid and subjected to end-imposed excitations[J]. International Journal of Non-Linear Mechanics, 2010, 45(7):667-680.

[12] Ai S, Xu Y, Kang Z, Yan F. Performance comparison of stress-objective and fatigue-objective optimisation for steel lazy wave risers[J]. Ships and Offshore Structures, 2019, 14(6):534544.

[13] Guo SX, Li YL, Li M, et al. Dynamic response analysis on flexible riser with different configurations in deep-water based on FEM simulation[C], OMAE 2018. V005T04A016.

[14] Jang H, Kim J W. Numerical investigation for vortexinduced vibrations of steel-lazy-wave-risers: Part II - CFD study on long flexible riser[C]. OMAE 2019. V002T08A031.

[15] Li Y, Guo S, Chen W. Analysis on multi-frequency vortexinduced vibration and mode competition of flexible deep-ocean riser in sheared fluid fields[J]. Journal of Petroleum Science \& Engineering, 2018, 163:378-386.

[16] Paz M. Structural dynamics. Theory and computation[M]. Van Nostrand Reinhold, 1980.

[17] José, Azcona, Xabier, et al. Experimental validation of a dynamic mooring lines code with tension and motion measurements of a submerged chain $[\mathrm{J}]$. Ocean Engineering, 2017,129:415-427. 